\title{
Apprendre et pratiquer sa créativité : des dispositifs en actes inspirés de la démarche des arbres de connaissances
}

Aude Ramseier et Sabine Oppliger, Haute école pédagogique du canton de Vaud (Suisse)

\section{Introduction}

Comment dans le cadre scolaire est-il possible d'encourager une culture de la créativité chez les élèves? Le terme de créativité associé à l'apprentissage est fréquemment utilisé, mais il ne suffit pas de vouloir « avoir » des élèves dits créatifs dans sa classe pour que les processus attendus se déroulent. De même, connaître les éléments qui peuvent stimuler la créativité chez les élèves en termes d'attitudes ou les obstacles qui peuvent la freiner ne garantit pas toujours son développement. Dans cet article, nous allons présenter des dispositifs et des démarches inédits qui ont été conduits dans le but de favoriser l'émergence de la créativité personnelle et collective des élèves dans une communauté d'apprenants (Brown et Campione).

\section{Contextualisation}

Une recherche-action a été mise sur pied par l'une d'entre nous dans sa classe d'élèves de neuf à onze ans dans le canton de Vaud en Suisse en vue d'expérimenter une démarche inspirée des arbres de connaissances (Authier et Lévy) durant l'année scolaire 2011-2012. Nous allons tout d'abord exposer les principaux axes qui constituent cette démarche puis les dispositifs utilisés, leur nature ainsi que les présupposés sur lesquels ils se fondent.

Le contexte dans lequel s'inscrit notre démarche repose principalement sur les principes de base des communautés d'apprenants tels que décrits par Ann L. Brown et Joseph Campione. Il y est relevé que pour créer un climat propice aux apprentissages souhaités, la posture de l'enseignant soit celle d'un accompagnateur de projets, ayant pour objectif de faciliter la mise en œuvre de travaux collectifs ou individuels, explicités et mis en lien avec des destinataires clairement identifiés. Les élèves sont placés dans une posture d'auteurs et d'acteurs de projets collectifs et personnels et co-créent ainsi la culture de la classe.

Ce contexte scolaire spécifique s'inspire également de la démarche des arbres de connaissances (abrégée dès lors ADC) telle que proposée par Michel Authier et Pierre Lévy. La philosophie intrinsèque à cette dernière stipule que « Chacun sait, on ne sait jamais, tout le savoir est dans l'humanité » (87). Elle insuffle des valeurs de reconnaissance et de partage des compétences personnelles et sociales. La démarche proposée pour les démontrer passe par la production de brevets qui représentent l'image des savoirs : «Ils reconnaissent des savoirs 
élémentaires, modulaires clairement identifiés. Ils sont attribués aux individus après passation d'une épreuve » (100).

L'usage d'un médiateur graphique sous forme d'arbre est utilisé pour mettre en évidence les différents brevets émis par les membres de la communauté au fur et à mesure de leur élaboration et ainsi faciliter l'acquisition de blasons qui représentent l'image de l'individu : «Il s'agit d'une représentation graphique des savoirs que l'individu possède y compris ceux qui sont nés de l'expérience, les « savoirs de vie » qui ne sont pas habituellement reconnus par des diplômes. Le blason évolue naturellement au fur et à mesure que l'individu acquiert ou parvient à faire reconnaître de nouveaux savoirs ». (100). Chacun a la possibilité à tout moment de rendre visible et partager une connaissance qui lui est propre (expertise) et également d'en acquérir de nouvelles proposées par les autres à travers ce dispositif médiateur.

En quoi ce dispositif des ADC a-t-il inspiré cette communauté d'apprenants et comment l'enseignante l'a-t-elle transposé dans sa classe ? Tout d'abord, elle a reformulé l'énoncé de base des ADC en l'adaptant à ce contexte d'apprentissage spécifique : « Chacun sait, personne ne sait tout, tout le savoir est dans la communauté ». Ensuite, la démarche d'Authier et Lévy a été adaptée à l'âge des enfants et aux contenus enseignés dans le canton de Vaud en Suisse. L'enseignante a conservé comme objectif la reconnaissance de savoirs globaux, le dépôt de brevets et l'arbre médiateur. Les élèves ont été stimulés tout au long de l'année scolaire à produire et présenter des projets sous la forme de textes, d'exposés et de jeux qui les autorisaient à exister pour une compétence à laquelle ils donnaient une valeur personnelle. L'évaluation des productions se faisait à travers les critères des compétences visées par le plan d'études en vigueur.

Un dispositif médiateur sous la forme d'un arbre papier a été présenté aux élèves comme étant un espace dans lequel ils allaient pouvoir faire apparaitre les savoirs dont ils étaient porteurs et ainsi se faire reconnaitre auprès de leurs pairs par ces connaissances variées (cf. Illustrations 1 et 2). Il leur a été expliqué que le but principal des productions qu'ils seraient amenés à effectuer en classe consistait en des projets de communication dont ils seraient les principaux destinataires. Selon le langage proposé par Authier et Lévy, il s'agit de déposer des brevets qui témoignent de leur expertise personnelle et qui font sens pour eux (cf. Illustration 4). Ils ont été invités à se mettre dans une posture d'auteur et d'illustrateur de leurs réalisations. Ces éléments faisaient intégralement partie des consignes explicites. Afin de mener à bien ces dépôts de brevets, l'importance des destinataires a été soulignée, des procédures d'écriture ont été institutionnalisées et différentes explicitations ont été apportées au sujet : 
- $\quad$ Des modalités plurielles de production individuelle et collective : il s'agissait de produire, seul ou à plusieurs, des brevets sous la forme de jeux, d'exposés libres et de devoirs principalement en mathématique et en français.

De l'entrainement à fabriquer ces brevets par désétayage progressif (Laveault), tout au long de l'année, le temps d'intégrer la complexité de la démarche de production. Tout d'abord, les enfants étaient invités à produire tous le même jeu (un mot croisé), puis ils ont pu en produire différents genres selon leur imagination et leur envie tout en respectant les critères formels propres à la production de projets (présence d'un titre, de consignes claires, etc.)

Des critères d'évaluation qui reposent sur les éléments formels sus-mentionnés.

Des moments d'échange et de régulation des brevets.

Des dispositifs de production de travaux et d'évaluation formative ont été engagés pour favoriser l'émergence de la créativité des élèves. Dans la partie suivante, nous allons présenter ces dispositifs ainsi que les présupposés sur lesquels ils se fondent.

\subsection{Les dispositifs en vigueur}

Trois dispositifs de nature différente ont été élaborés dans la classe. Premièrement, un médiateur sous la forme d'arbre qui sert à mettre en évidence les règles, les valeurs, les rôles et les productions de la communauté. Ce médiateur n'a pas seulement pour but de faire apparaître les savoirs. Afin de favoriser un climat de classe riche en interactions positives et une dynamique du groupe favorable aux apprentissages, l'enseignante a choisi d'expliciter le plus possible les normes en vigueur avec les élèves pour assurer l'équité, le sentiment de confiance et de sécurité nécessaires à la posture d'apprenant, et d'autant plus à celles d'auteur et de créateur. Deuxièmement, des dispositifs d'évaluation formative qui permettent de réguler les apprentissages sociaux et cognitifs. Ils existent sous la forme d'un conseil de classe hebdomadaire et de différents dispositifs d'auto-évaluation et de co-évaluation des productions. Ils permettent de réguler l'acquisition des normes en vigueur ainsi que les apprentissages. Troisièmement, le dispositif d'apprentissages sous la forme de brevets qui encourage à la reconnaissance et au partage des compétences spécifiques à chacun. Il donne également un but et un sens aux apprentissages des élèves, incite à les canaliser dans des projets concrets avec des destinataires bien définis favorisant ainsi leur motivation.

Ces dispositifs orientent l'enseignement et la posture de l'enseignant. Les intentions qui en découlent s'appuient sur des valeurs de partage qui sont en résonnance avec celles promues par la pédagogie coopérative (Howden et Kopiec). «Tapies dans l'ombre de nos enthousiasmes, de nos doutes ou de nos remises en question : les valeurs. Celles-ci méritent notre attention, sur le plan individuel et collectif, car elles fondent et orientent tant la conduite 
de l'enseignant dans sa classe, jour après jour, que le système éducatif dans son ensemble (les finalités de l'éducation). Ingrédients à la base de nos croyances et de nos représentations, inspiratrices des nombreuses décisions à prendre, les valeurs sont le moteur de l'engagement professionnel ». Cette citation extraite de l'article «Les valeurs au cœur de la profession enseignante ! » rédigé en 2013 par Eric Walther, mais non publié, propose une définition qui convient particulièrement à ce contexte.

Ces valeurs sont les suivantes : la confiance, l'ouverture envers les autres, l'entraide, l'égalité, le droit à l'erreur, la solidarité, l'engagement, le plaisir et la synergie sont clairement formulés et travaillés avec les élèves durant toute l'année scolaire (cf. Illustration 3). Elles sont particulièrement importantes au même titre que les règles de classe pour la constitution de la norme, car elles préservent et maintiennent un climat propice aux apprentissages coopératifs et aux relations entre les apprenants. Autant les règles de classe sont-elles explicitées pour réguler les comportements et les interactions et maintenir ainsi l'équité de traitement au sein du groupe, autant l'explicitation des valeurs garantit-elle à l'individu une équité dans le traitement qui touche à l'ensemble de ses actions.

\section{2. Question de recherche et hypothèse}

Dans le cadre de cet article, nous allons questionner comment et en quoi cet environnement est porteur de conditions propices à la créativité. Les trois dispositifs décrits précédemment visent à placer les élèves dans une position d'auteurs-acteurs qui nous amène à poser la question de recherche suivante : en quoi les dispositifs mis en actes proposent des conditions-cadres favorables à l'émergence de la créativité des élèves ? Nous faisons l'hypothèse que des dispositifs de cette nature encouragent le développement d'une créativité partagée dans la classe.

\section{Cadre conceptuel pour la mise en ouvre des dispositifs}

Dans ce contexte d'apprentissage spécifique, la créativité est abordée comme un concept transversal qui se déploie de manière individuelle ou collective au travers du dispositif médiateur de l'ADC, des brevets ainsi que des dispositifs d'évaluation formative et qui peut être définie comme étant « la capacité à réaliser une production qui soit à la fois nouvelle et adaptée au contexte dans lequel elle se manifeste » (Lubart 10).

Les recherches de Todd Lubart et plus particulièrement son approche d'une créativité dite multivariée mettent en évidence que la créativité n'est pas seulement une caractéristique cognitive, mais le produit de l'interaction entre des composantes conatives (conduites conscientes en termes d'efforts qui se réfèrent au traits de la personnalité, mais aussi à la 
motivation), des composantes affectives (émotions, préférences, intérêt) et des composantes relatives à l'environnement. C'est « la combinaison de ces multiples facteurs qui influencera le potentiel créatif de chaque individu, son développement, ainsi que son expression» (Besançon et Lubart 4).

Une production créative nécessite non seulement une succession d'actes cognitifs, tant dans les pensées que dans les actions, mais est également dépendante de plusieurs facteurs qui entrent en interaction, notamment « la pensée divergente-exploratoire qui se réfère à la capacité à rechercher des idées, à puiser dans son environnement et ses connaissances des éléments en lien avec la tâche » (4). Cette citation rappelle que l'environnement a un rôle à jouer dans la mise en condition d'une posture de recherche chez l'apprenant.

Liée aux facteurs conatifs, la motivation est essentielle pour qu'un sujet s'engage dans une tâche ou un projet qui a du sens pour lui. Elle peut être intrinsèque et prendre naissance dans les besoins de l'individu (par exemple, la curiosité, l'envie d'exprimer son individualité) ou extrinsèque car générée par des incitations extérieures, comme la reconnaissance sociale par des pairs (Amabile). L'influence suscitée par l'environnement sur les productions créatives est indubitable, il peut être « source de stimulation sociale, d'opportunités et de leviers, mais aussi de freins, d'inhibition et de contraintes pour la créativité » (Besançon et Lubart, 4).

Les facteurs émotionnels (l'attention portée aux émotions vécues, leur intensité) sont également présents en orientant ou en nuançant la pensée associative lors de la recherche d'idées, notamment, comme le relèvent ces auteurs, l'idiosyncrasie qu'ils définissent comme la réaction, le tempérament et la manière de se comporter propre à chaque individu face aux influences de l'environnement (physique et personnel). En lien avec cette composante affective, Mihaly Csikszentmihalyi met en évidence les besoins d'une personne créative en prenant en compte deux dimensions :

- Une première externe liée au besoin de l'autre, du dehors qui permet de répondre aux besoins de retour, de résistance, de se reconnaître, de s'identifier, de se situer par rapport à son environnement et de s'inscrire dans un contexte.

- Une seconde qui a trait au besoin de pouvoir se concentrer sur soi-même avec notamment le fait de se confronter avec sa propre conception du monde, d'inscrire le nouveau dans son propre univers, de sentir ses envies et ses besoins, de se sentir dans un processus, de pouvoir essayer, échouer, réessayer et de trouver de nouvelles configurations à l'intérieur de son univers.

Notre analyse montrera comment les dispositifs prévus dans la démarche inspirée des ADC permettent la prise en compte de ces doubles besoins en favorisant l'émergence des 
processus créatifs chez les élèves.

Nous reviendrons aussi de manière plus détaillée sur les quatre facettes dont Pasi Sahlberg envisage la notion de créativité. La première touche au fait de concevoir une production nouvelle qui a du sens dans tous les contextes d'activité. La deuxième postule que la créativité peut être à la fois individuelle et collective, en pensée comme en action. La troisième relève que le processus créatif devrait engager des applications délibérées de savoirs, de compétences et d'attitudes antérieurs. La quatrième rappelle que chacun possède quelque capacité créative dans un domaine.

\section{Aspects méthodologiques et recueil de données}

Durant l'année scolaire 2011-2012, dans une classe du canton de Vaud composée de vingt-six élèves de neuf à onze ans, une recherche-action mise sur pied en vue d'observer le processus d'évolution de la démarche inspirée des ADC (Authier et Lévy) a fait l'objet de différentes observations.

Pour cet article, nous avons choisi de focaliser notre attention sur les conditions-cadre de la démarche en les analysant du point de vue de la créativité. Nous avons privilégié une approche de type qualitatif qui porte sur l'analyse des intentions liées aux dispositifs en place et sur des bilans auto-évaluatifs (cf. Illustrations 5 et 6) réalisés en fin d'année scolaire par les élèves. Ces données ont été traitées en regard de notre questionnement et de notre cadre théorique.

\section{Analyse}

\subsection{Un contexte motivant, tremplin pour les processus créatifs.}

Les dispositifs de la démarche des ADC remplissent plusieurs préalables mentionnés par des auteurs (Csikszentmihalyi; Jaoui; Lubart; Sahlberg), comme nécessaires à l'émergence de la créativité tels que légitimer le droit à l'erreur (valeur clairement explicitée), encourager la pluralité des réponses dans le contexte scolaire, favoriser un va-et-vient continu entre l'individu qui crée et le groupe en prenant en compte ses besoins internes et externes et pratiquer de nombreuses rétroactions positives pour établir un espace sécurisant (dans le cadre du conseil de classe). Les conditions mises en place engagent les élèves dans une démarche concrète et adaptée : l'instauration progressive d'un cadre précis avec des normes sécurise les élèves et leur permet d'expérimenter et de s'exprimer en autorisant par exemple l'entraide, le droit à l'erreur, les attitudes de non-jugement, l'engagement, l'ouverture aux autres, etc.

L'enseignant leur attribue une posture d'auteur-acteur propre à la communauté 
d'apprenants et propose des modalités d'enseignement/apprentissage qui sortent de l'ordinaire. Durant toute l'année scolaire, ils sont sollicités pour concevoir et réaliser des brevets sous forme de projets de communication. Les consignes invitent les élèves à puiser dans leur imagination et leur expérience pour réaliser des travaux qui engagent leurs connaissances en les réactualisant. Les projets ainsi conçus témoignent des savoirs acquis et des processus d'apprentissage, mais aussi de manière plus large de leur intégration en tant qu'élèves au sein de la communauté-classe qui répondent aux objectifs fixés par le plan d'étude en vigueur.

En lien avec les quatre facettes relevées par Sahlberg, la question du sens est un incontournable dans la démarche des ADC, tant dans ses fondements que dans ses visées. Les apprenants s'engagent dans des tâches qui requièrent des processus créatifs, mais qui répondent aussi à des intérêts de connaissances et de partage. Les élèves choisissent le sujet, la manière de l'élaborer, de le présenter et personnalisent sa forme finale.

Le potentiel créatif des élèves est activé de manière individuelle et/ou collectivement par des travaux en duos ou triades et ceci tant dans des productions qu'au niveau des pensées par le partage de valeurs communes. Lorsqu'ils créent des jeux avec leurs pairs, ils mettent en commun des savoirs, des moyens et des ressources qui se complexifient et s'étayent mutuellement, mais aussi le fait de «co-créer»concrétise les valeurs promues dans le dispositif.

Le fait de mettre en pratique ces valeurs de façon répétée génère une mise en confiance durable et propice à l'émergence et au développement des processus créatifs chez les élèves. La dimension affective liée aux besoins de sécurité et de reconnaissance est dépendante de l'explicitation et de la clarification du cadre en termes de règles, de valeurs, d'intentions. En lien avec les doubles besoins présents dans l'acte créatif mis en évidence par Csikszentmihalyi, la métaphore de l'arbre de connaissances comme dispositif médiateur prend tout son sens, par exemple quand les élèves font l'expérience de l'intégration progressive des attentes du cadre, explicitées et co-construites qui protègent les débuts en créativité souvent fragiles et les aident à surmonter les résistances dues aux jugements de valeurs.

\subsection{La posture de l'enseignant dans l'émergence de la créativité des élèves}

Les conditions favorables sous-entendent également que l'enseignant assume la responsabilité de jouer un rôle actif dans le développement de la créativité de ses élèves par sa posture d'ouverture à cette dimension et qu'il l'intègre de manière intentionnelle et transversale dans son enseignement. Ceci se traduit par l'explicitation de valeurs, le partage des savoirs et des apprentissages, la co-construction d'une culture de classe et de situations d'apprentissages qui permettent aux élèves de faire l'expérience d'une créativité partagée (Berthod). En variant 
les moyens, les supports, les finalités, ils sont capables de mieux utiliser leurs potentialités et améliorent le niveau de leurs créations. Elaborer des jeux, par exemple, est un apprentissage. Au fur à mesure de l'année scolaire, sont intégrés les procédés, les techniques, l'utilisation des matériaux, la confiance dans la valeur du travail et des échanges pour co-construire, mais également dans ses propres aptitudes à persévérer, à inventer et donner forme.

La créativité des élèves est aussi stimulée par un certain nombre de contraintes identifiées et connues par la classe par exemple les critères qui composent l'auto ou coévaluation formative. Cependant, les activités pour concevoir les projets de communication sont basées sur des consignes suffisamment ouvertes dans leur formulation pour permettre aux élèves de donner des réponses personnelles et originales. Nous allons maintenant les analyser au travers de leurs productions.

\subsection{Une imagination matérialisée en brevets avec la mise en « jeux » et en « je »} des apprentissages

La diversité des thématiques choisies par la communauté d'apprenants témoigne d'une créativité en actes. Elle traite de sujets variés tels que :

- La nature et les phénomènes naturels : «L'arbre magique », les pivoines, les étangs, les geysers, les fossiles, ...

- $\quad$ Les animaux de compagnie et leur environnement : le lapin, la tortue, l'aquarium, ...

- La santé, la diététique et les arts de la table : «Mon assiette », plusieurs recettes de cuisines, etc.

- $\quad$ La musique : style de musique «boogie woogie », présentation d'un compositeur, d'un instrument, d'un morceau de musique, etc.

- $\quad$ La famille et des événements existentiels : la maladie, la mort, etc.

- Des livres de jeux et de nombreux exercices en français de type «phrases à trous », «phrases codées », « mots cachés », « mots croisés » ou en mathématiques.

La mise en œuvre et la forme finale des projets de communication indiquent que les élèves se sont approprié la démarche. Ils se sont clairement identifiés sur la page de couverture comme auteur ou illustrateur de leurs histoires. Ils ont utilisé le «je » pour présenter un intérêt de connaissance. Ils ont soigné leur mise en page en y ajoutant des éléments esthétiques. Leurs brevets sont enrichis de dessins originaux ou d'images judicieusement choisies.

La multiplicité des réponses apportées par les élèves est aussi un bon indice, en termes de créativité, de la pertinence des types de sollicitation faites par l'enseignante et suscitées par la démarche inspirée des ADC. A partir d'une même consigne «réaliser un projet de communication en français », les thématiques sont non seulement très riches mais aussi traitées 
sous des formes différentes : histoires inventées, dossiers écrits, exposés oraux. La pluralité des moyens choisis par les élèves témoigne d'une singularité assumée et capable de « diverger » pour élaborer un projet qui fait sens pour soi, avec aussi des résultats intéressants en termes de fluidité ${ }^{1}$ et d'élaboration ${ }^{2} »$. $^{2}$

Les élèves développent une relation vivante et dynamique aux apprentissages et aux savoirs. Ils mentionnent qu'ils comportent des pièges, des dangers. De plus, les règles qui accompagnent les jeux, rendent attentifs leurs camarades aux nombreux risques possibles présents, mais les créateurs stimulent leurs pairs à se lancer dans l'épreuve à l'aide de renforcements positifs tels que : «Vas-y!», «Courage... ». Quand ils rattachent les brevets aux valeurs, ils tissent un lien explicite entre le processus et le produit, entre l'expérience du processus et du produit, à savoir sa mise en acte dans la réalisation des brevets.

\section{Conclusion et perspectives}

Nous nous demandions en quoi les dispositifs de la démarche inspirée des ADC favorisent l'émergence de la créativité chez les élèves. Les bilans auto-évaluatifs laissent apparaître que les valeurs proposées par le dispositif médiateur ont été investies par les élèves et rattachées à des objets de productions concrets. Les apprenants ont fait l'expérience d'une culture de créativité partagée tout au long de l'année qui institutionnalise cette dimension en l'intégrant dans un processus continu qui la légitime comme objet d'apprentissage. Les élèves font l'expérience que la créativité n'est ni mystérieuse, ni magique et que l'environnement, par le biais de l'enseignant, a un rôle actif à jouer pour la promouvoir et la développer dans sa classe.

Les dispositifs mis en place issus de la philosophie insufflée par les ADC reformulés par l'enseignante « chacun sait, personne ne sait tout, tout le savoir est dans la communauté » instaurent des valeurs et une culture de reconnaissance par la création d'espaces qui la rendent possible. Ces éléments officialisés, dès le début de l'année scolaire, par le dispositif médiateur «arbre », rendent légitime leur expression au travers des différents brevets. Les conditionscadres prennent en considération les temps d'intégration et de régulation et permettent aux élèves de développer un sentiment d'appartenance au groupe en s'appropriant des éléments liés au prescrit et d'autres issus des interactions qui font de cet espace le lieu unique de l'expérience. Les apprenants sont encouragés à endosser la posture d'auteur-acteur et à mener des projets qui font sens pour eux.

L'enseignant par sa posture d'accompagnateur facilitateur est le garant de la conduite de cette démarche motivante qui éveille des intérêts, offre des espaces d'expérimentation en 
lien direct avec cette culture de reconnaissance. Les dispositifs de l'arbre médiateur mobilisent les potentialités individuelles au service du collectif et de la co-création d'une culture de la reconnaissance des compétences des élèves en classe.

\section{Bibliographie}

Amabile, Teresa M. Creativity in Context : Update to " the Social Psychology of Creativity ». Boulder, CO : Westview, 1996.

Authier, Michel et Pierre Lévy. Les arbres de connaissances. Paris : La Découverte, 1996.

Besançon, Maud et Todd Lubart. «Favoriser la créativité des élèves par une approche multivariée ». Educateur 2 (2014) : 3-5.

Berthod, Eric. «La créativité, une compétence en partage ». Résonnances. Mensuel de l'école valaisanne 8 (2014) : 6-7.

Brown, Ann L. et Joseph Campione. «Concevoir une communauté de jeunes élèves, leçons théoriques et pratiques ». Revue française de pédagogie 111 (1995) : 11-33.

Csikszentmihalyi, Mihaly. La créativité : psychologie de la découverte et de l'invention. Paris : Laffont, 2006.

Howden, Jim et Marguerite Kopiec. Structurer le succès : un calendrier d'implantation de la coopération. Montréal/Toronto : La Chenelière/McGraw-Hill, 2006.

Jaoui, Hubert. La créativité. Le trésor inconnu. Paris : Morisset, 1995.

Laveault, Dany. «De la 'régulation' au 'réglage' : élaboration d'un modèle d'autoévaluation des apprentissages ». Régulation des apprentissages en situation scolaire et en formation. Dir. Linda Allal et Lucie Mottier Lopez. Bruxelles : De Boeck, 2007. 207234.

Lubart, Todd. Psychologie de la créativité. Paris : Colin, 2003.

Sahlberg, Pasi. « Creativity and Innovation through Lifelong Learning ». Lifelong Learning in Europe 16 (2011).

\footnotetext{
NOTES

${ }^{1}$ Aptitude à produire un grand nombre d'idée ou de réponses à partir d'une question donnée et qui est associée à la pensée dite divergente.

${ }^{2}$ Aptitude à développer, structurer, préciser, affiner une réponse au moment où sont considérées les idées trouvées et sélectionnées ainsi que les meilleures solutions selon différents critères de choix qui apparaissent dans la phase de la pensée dite convergente.
} 
Annexes : Illustrations du dispositif de l'arbre de connaissances réalisé dans une classe, volée 2011-2012 (photos A. Ramseier)

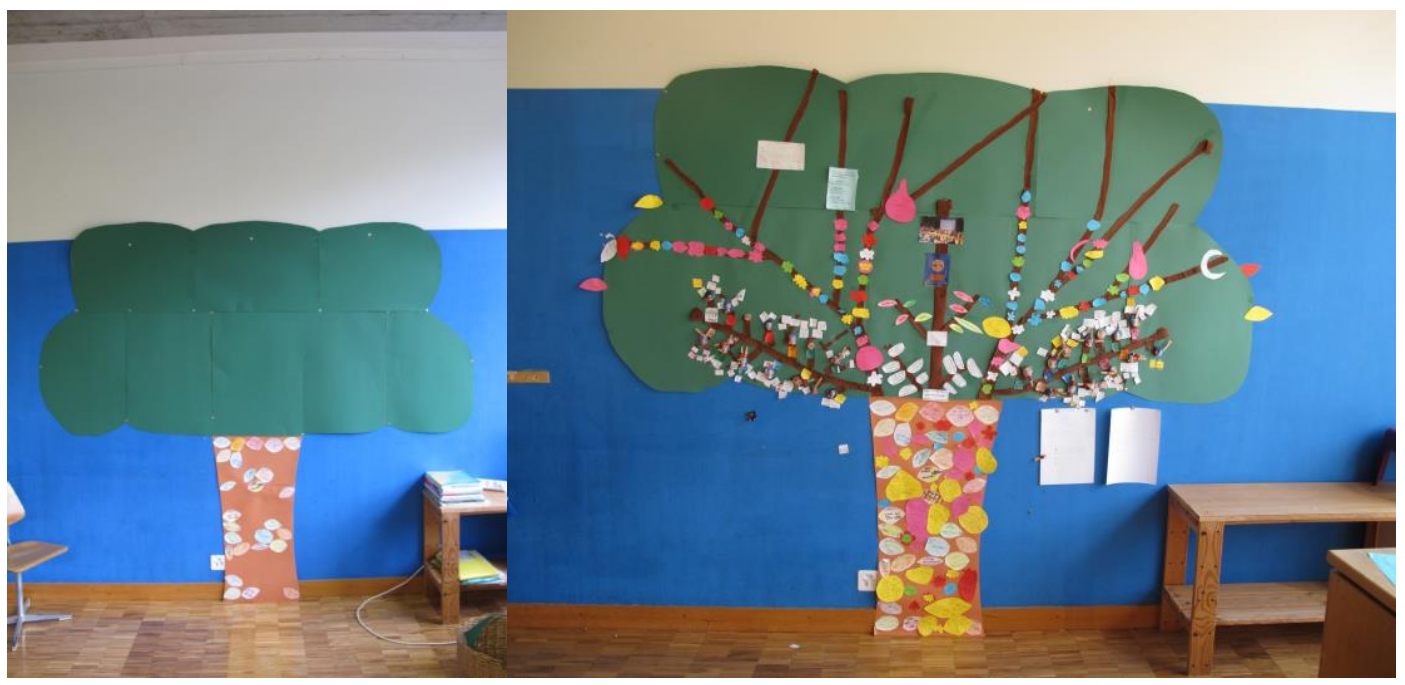

Illustrations 1 et 2 : Evolution du dispositif de l'arbre de connaissances de la rentrée scolaire en août 2011 à juin 2012.

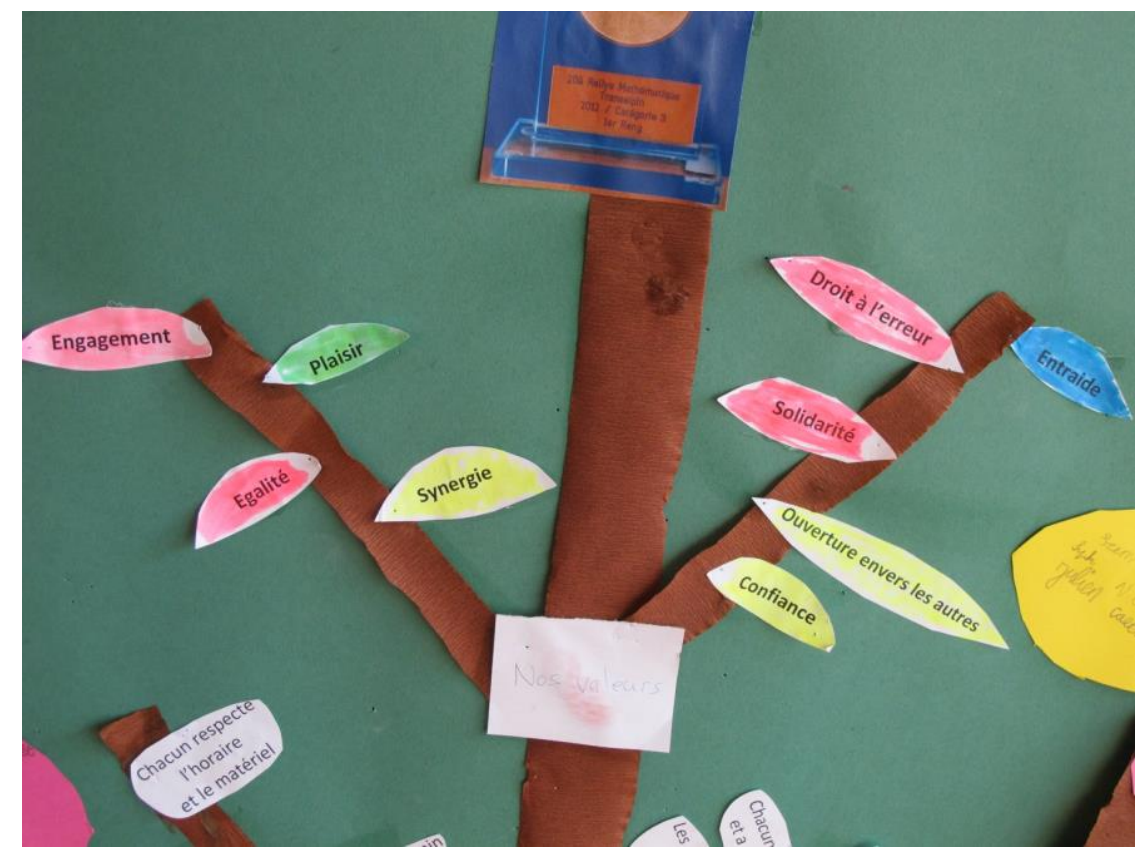

Illustration 3: Les valeurs coopératives (Howden et Kopiec) constitutives du dispositif médiateur de l'arbre de connaissances 


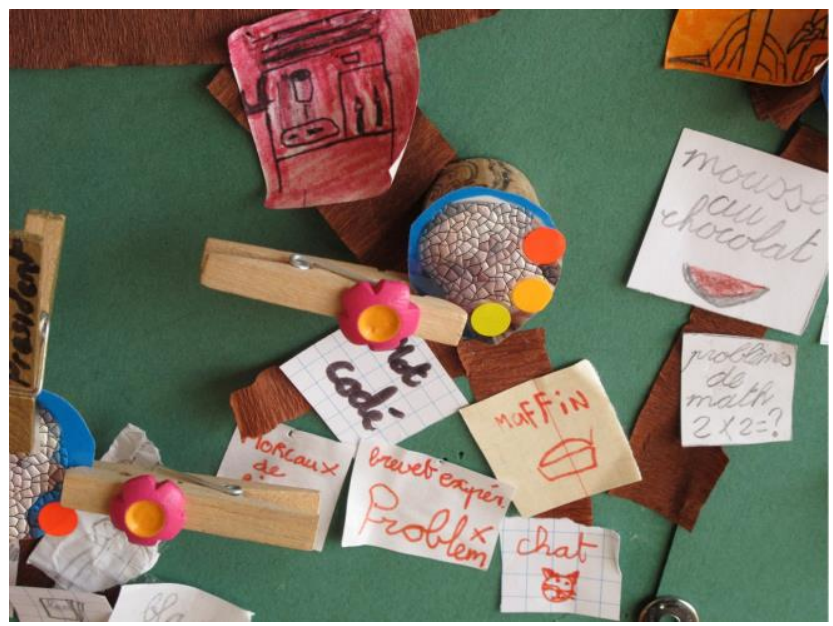

Illustration 4 : Les expertises des élèves apparaissent sous forme de brevets dans le dispositif des arbres de connaissances
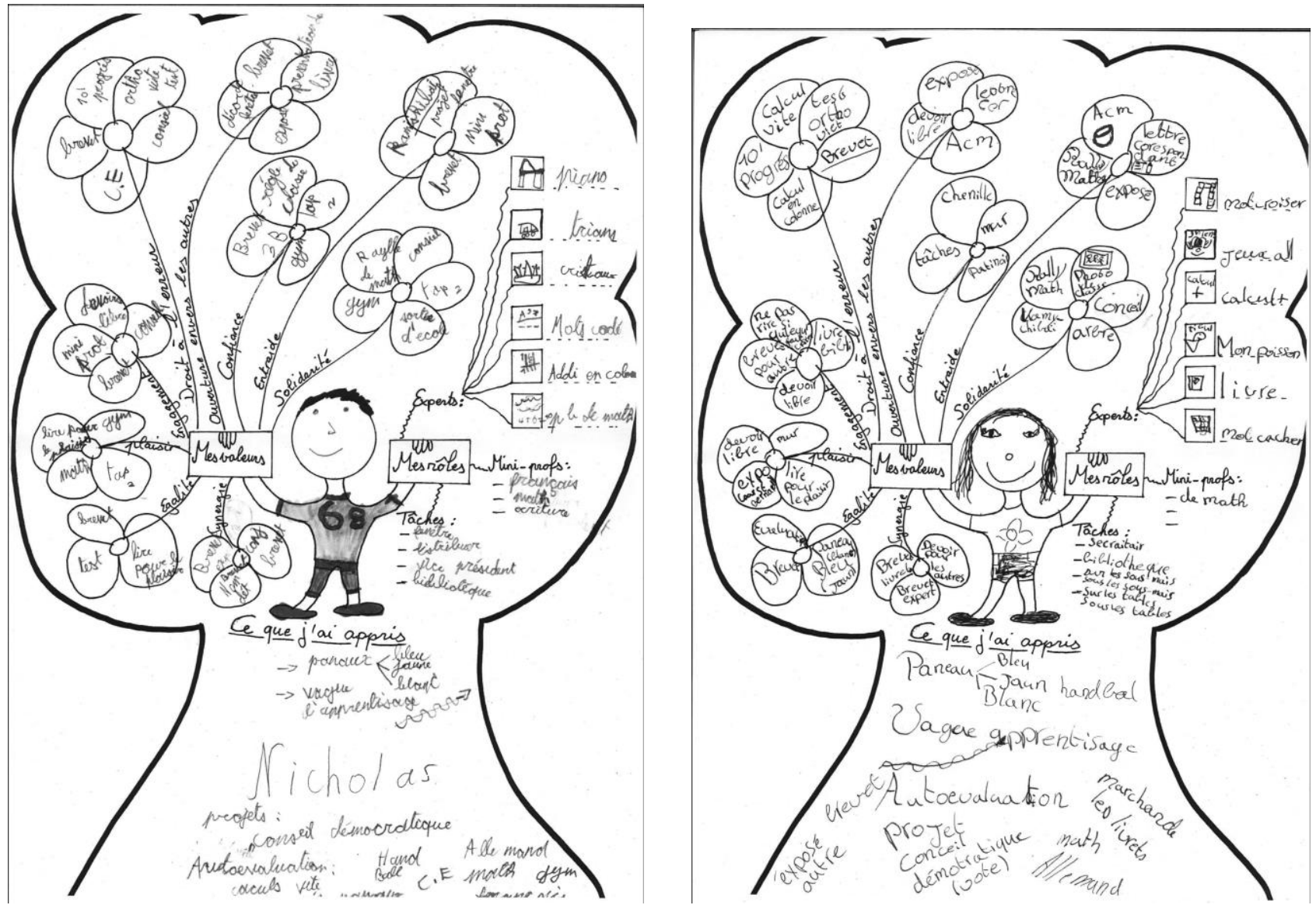

Illustrations 5 et 6 : Bilans auto-évaluatifs réalisés par les élèves sur leur apprentissage en lien avec les valeurs explicitées sur le dispositif médiateur arbre 